\title{
Effects of Rest Interval on Strength Recovery in Breast Cancer Survivors
}

Authors

Affiliations
C. A. Vieira ${ }^{1}$, C. L. Battaglini ${ }^{2}$, J. B. Ferreira-Junior ${ }^{3}$, A. Vieira ${ }^{3}$, M. F. Brito Vogt $^{4}$, R. Freitas-Junior ${ }^{5}$, R. J. Oliveira ${ }^{3}$, M. Bottaro

Affiliation addresses are listed at the end of the article
Key words

strength recovery

- muscle fatigue

0 isokinetic accepted after revision December 11, 2014

\section{Bibliography} DOI http://dx.doi.org/ 10.1055/s-0034-1398579 Published online:

March 17, 2015

Int J Sports Med 2015; 36: 573-578 @ Georg Thieme Verlag KG Stuttgart · New York ISSN 0172-4622

\section{Correspondence}

Prof. Carlos Alexandre Vieiro College of Physical EducationFederal University of Goias Campus Samambaia Goiania 74001-970 Brazil

Tel.: + 55/623/5211 141 Fax: + 55/623/5211 141 vieiraca1@uol.com.br

\section{Abstract \\ $\nabla$}

The purpose of this study was to compare the acute effect of 2 different resting intervals (RI) between sets of isokinetic knee extension exercise on peak torque (PT) and total work (TW) in breast cancer survivors (BCS) and control group (CNT). 16 BCS (52 \pm 4 years) and 14 CNT (53 \pm 6 years) performed 3 sets of 10 unilateral isokinetic knee extension repetitions at $60^{\circ} . \mathrm{s}^{-1}$ on 2 separate days with 2 different RI between sets ( 1 and $2 \mathrm{~min}$ ). There was a significant interaction between groups vs. exercise sets $(p=0.03)$ and $R I$

\section{Introduction}

Resistance training has been widely accepted as the most popular exercise training mode for increasing muscular strength, endurance, hypertrophy and muscular power [18]. For efficient and safe training it is important to design resistance training programs based on scientific evidence. In order to maximize strength training benefits, the empirically based manipulation of acute training variables including intensity, frequency, volume, exercise choice, rest interval (RI) and exercise order $[32,33,38]$ are critical. Many studies have been conducted to evaluate the effects of different rest intervals between sets on acute responses and chronic adaptations of resistance training $[9,11,12,18,32,33,38,40]$. Adequate between-sets resting periods are necessary for appropriate muscle recovery to mitigate the effects of fatigue, and thus properly achieve increases in training volume [32,33]. Several studies have compared RI between sets not only in younger but also in special populations, such as children, young adults and the elderly $[8,9,12]$. It has been reported that older men are more fatigue-resistant than younger adults $[6,7,9,22-24]$. Further, younger women vs. exercise sets $(\mathrm{p}<0.001)$ for PT. PT was greater in CNT at $1^{\text {st }}$ and $2^{\text {nd }}$ sets compared to BCS group (CNT, $133.4 \pm 20.8$ and BCS $107.6 \pm 19.9 \mathrm{Nm}$, $\mathrm{p}=0.012$ and CNT, $118.9 \pm 19.6$ and BCS, $97.1 \pm 15.9 \mathrm{Nm}, \mathrm{p}=0.045$, respectively). The TW of the knee extensor was significant greater in CNT than BCS group for all 3 knee extension exercise sets. In conclusion, the present study suggests that Breast Cancer Survivors women may need a longer rest interval (longer than $2 \mathrm{~min}$ ) to be able to fully recover during a 3 sets of isokinetic knee extension exercise training session.

have greater resistance to fatigue than men $[13,31]$. In addition, older women seem to be more fatigue-resistant than older men $[6,21]$. Theou et al. [40] found that older women required a 1:1 exercise-to-rest ratio for knee flexor recovery, while young women required a longer 1:2 exercise-to-rest ratio. In addition, Bottaro et al. [9] reported a significant decline in PT when 1 and 2 min rest intervals were used for young men, but not when a 2 min rest interval was applied for elderly men. They reported that young men need longer RI because they produce more force and the resistance to fatigue is inversely related to force production.

Cancer-related fatigue (CRF) is a very common side effect experienced by breast cancer survivors who underwent chemotherapy and/or radiation therapy [16]. CRF is a multifactorial process involving cancer treatment-related negative alterations to many physiological systems, sleep disturbances, and dietary intake alterations. All these are factors that can influence the commonly observed reduction in overall physical activity levels, leading to a downward spiral of reduced physical functioning and worsening of fatigue [3]. Regular exercise has been shown to be an intervention that can disrupt this down- 
ward spiral cycle of overall physical decline and debilitating fatigue [3]. Furthermore, significant benefits including improvements in cardiopulmonary function (6-39\% post training) as well as increases in muscle strength (11-110\% post training) [17] have been observed in different cancer populations, and these improvements have been associated with reduced overall physical dysfunction and fatigue levels [3]. Thus, since resistance to fatigue may influence rest periods during exercise, cancer survivors may experience different rest intervals between sets during resistance exercise. Therefore, evaluating training variables included in a session of strength training, most specifically the resting periods necessary for optimizing training responses between exercise sets, is fundamental for the design of more efficacious resistance training programs in cancer patients.

The purpose of this study was to compare the acute effect of 2 different RI between sets of knee extension exercise on muscle strength and muscle work capacity in breast cancer survivors. It was hypothesized that PT and TW in BCS would be lower than in the CNT group, and $1 \mathrm{~min}$ RI would be sufficient to recover PT and TW in the BCS group. The rationale for our hypothesis is that the RI required is dependent on muscle strength produced [9]. The results of this study may serve as a stepping-stone for future research in the area of the effects of resistance training in breast cancer survivors, so that more appropriate and effective exercise training interventions using resistance training can maximize training response.

\section{Methods}

$\nabla$

\section{Experimental procedures}

To test the effect of RI on peak torque (PT) and total work (TW), both the breast cancer survivors (BCS) and control group (CNT) performed an isokinetic knee extension exercise training session with 2 different RI ( 1 and $2 \mathrm{~min}$ ) between sets on 2 separate days. Participants were randomly assigned to perform either the 1 or 2 min RI trials based on the website randomization.com (http://www.randomization.com). The 2 exercise training sessions were conducted with a minimum of $72 \mathrm{~h}$ and a maximum of 1 week between each session. The resting periods and the number of sets were selected following recommendations by the American College of Sport Medicine (ACSM) for resistance training designed for the development of muscle strength [34]. The total volume of training used in this study was based on previous studies conducted with elderly people [40] with the velocity of exercise set at $60^{\circ} . \mathrm{s}^{-1}$, a speed often used for the isokinetic testing in both young [41] and older adults $[11,40]$. The decline of torque between exercise sets, which has been commonly used to quantify muscle fatigue in isokinetic testing of older people [19], was used for quantification of muscular fatigue during the exercise sessions. To avoid circadian influence, participants performed under both conditions at the same time of day.

\section{Participants}

All participants were informed of the purpose, procedures, possible discomforts, risks and benefits of the study prior to signing an informed consent form approved by the University Institutional Ethics Committee (Protocol: 126/12). The study was performed in accordance with the ethical standards of this journal [20]. The inclusion criteria for participation in the study for the
BCS group included: age-range 44-65 years, post-menopausal, sedentary (not involved in any regular exercise training within the past 12 months), cancer therapy completion, including surgery, radiotherapy, and/or chemotherapy, and were at least 6 months post-treatment. For the CNT group, the criteria for inclusion included: age-range 44-65 years, post-menopausal, sedentary, and sufficiently healthy to participate in resistance training. The criteria for determining post-menopausal status for both groups followed recommendations by the World Health Organization [29]. Participants were excluded from this study if diagnosed with lymphedema (BCS group), advanced hypertension, cardiovascular disease, and/or any orthopedic limitation that would preclude the ability to complete the study protocol.

\section{Familiarization and warm-up}

As part of familiarization with isokinetic knee extension and warm-up, volunteers performed 2 sets of 5 knee extension repetitions at $60^{\circ} . \mathrm{s}^{-1}$ with $1 \mathrm{~min}$ rest between sets [30]. $3 \mathrm{~min}$ after the familiarization/warm-up session, volunteers performed either the 1 or 2 min RI exercise training sessions. Both days of testing followed the exact same format.

\section{Measurement of isokinetic torque}

The isokinetic training session consisted of 3 sets of 10 unilateral isokinetic knee extension (right leg) repetitions at $60^{\circ} . \mathrm{s}^{-1}$. Isokinetic PT, as well as TW during knee extension were measured using a Biodex System 3 Isokinetic Dynamometer (Biodex Medical, Inc., Shirley, NY, USA). Subjects were positioned comfortably on the dynamometer seat with safety belts fastened to the trunk, pelvis and thigh to minimize extraneous body movements which could affect the PT and TW output values. The lateral epicondyle of the femur was used as a marker to align the knee rotation axis and the instrument rotation axis, allowing free and comfortable knee flexion and extension from $80^{\circ}$ flexion to full extension. With the subjects positioned on the seat, the following measures were recorded: seat height, backrest inclination, dynamometer height and lever arm length. These measures were recorded during the familiarization session and standardized as the test position for both testing sessions. Gravity correction was obtained by measuring the torque exerted by the lever arm and the subject's leg at full extension as well as in a relaxed position. The values of the isokinetic variables were automatically adjusted for gravity with the software Biodex Advantage (Biodex Medical, Inc., Shirley, NY, USA). All these procedures have been previously described [9].

The calibration of the Biodex dynamometer was carried out according to the specifications contained in the instruction manual. For the test, the subjects were asked to cross their arms across the chest [39]. During the tests, subjects received verbal encouragement throughout the session. The same tester carried out the test procedures for all subjects.

\section{Statistical analysis}

Data are presented as mean \pm standard deviation. The Kolmogorov-Smirnov test was used to determine whether the sample data was normally distributed. A 3-way mixed model ANOVA [Group (BCS and CNT) vs. Rest Interval ( 1 and $2 \mathrm{~min}$ ) vs. set ( $1^{\text {st }}$, $2^{\text {nd }}$ and $\left.3^{\text {rd }}\right)$ ] was used to analyze knee extension PT and TW. In the case of significant differences, a LSD post hoc test was used. Significance level was set a-priori at $\mathrm{P}<0.05$. 


\section{Results}

$\nabla$

The BCS group was selected from 111 medical histories from the University Hospital files. After phone contact, 31 BCS volunteered to participate in the study protocol. However, only 19 BCS met the inclusion criteria and 16 finished the study protocols. The CNT group was selected at random from the respondents to fliers distributed to social clubs, public offices, and by word-ofmouth. 25 women volunteered to participate, and 14 met the inclusion criteria and finished the study protocol ( $\bullet$ Fig. 1 for Flow of participants enrollment in the study). Participant characteristics are presented in $\odot$ Table 1 .

The BCS group participants were $16.2 \pm 6.77$ months post-treatment. 11 women in the BCS group had undergone surgery, chemotherapy and radiation therapy, 3 had undergone surgery and chemotherapy, and 2 had surgery and radiation therapy. All BCS were undergoing hormonal therapy with tamoxifen during the study.

PT and TW for the BCS and CNT group in both 1 min and 2 min RI sessions are presented in $\bullet$ Table 2.

There were no significant interactions between groups vs. rest interval vs. exercise sets for knee extension PT $(p=0.41)$ or TW $(p=0.62)$. However, there was a significant interaction between groups vs. exercise sets $(p=0.03)$ and rest interval vs. exercise sets $(p<0.001)$ for PT. PT was significantly higher in the CNT group in the $1^{\text {st }}$ and $2^{\text {nd }}$ set compared to BCS group ( $\mathrm{p}=0.012$ and $\mathrm{p}=0.045$, respectively). PT was also higher in 2 min rest interval condition at $2^{\text {nd }}$ and $3^{\text {rd }}$ set compared to 1 min rest condition for both groups $(\mathrm{p}<0.001)$. In addition, PT significantly decreased throughout exercise for both groups in both RI $(\mathrm{p}<0.05)$.

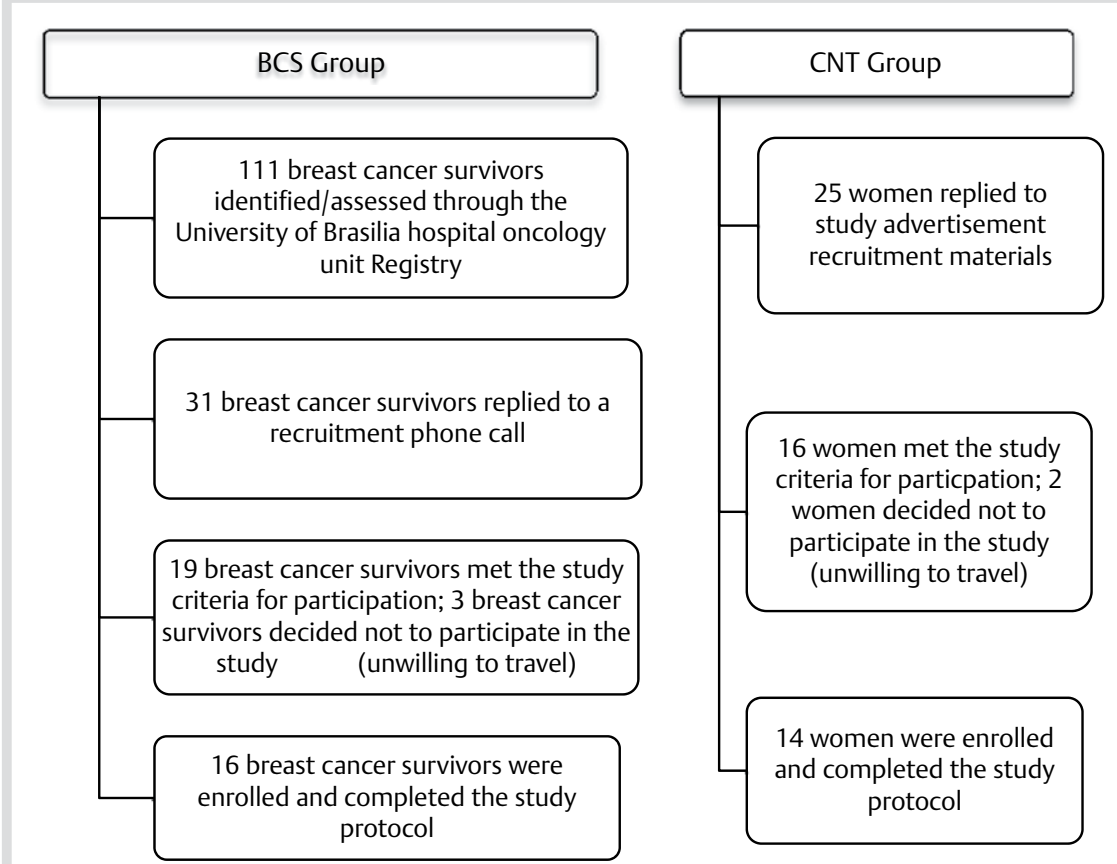

Fig. 1 Flow of participant enrollment in the study.

Table 1 Participants characteristics.

\begin{tabular}{|c|c|c|c|c|c|}
\hline Groups & $\begin{array}{l}\text { Age Mean } \pm S D \\
\text { (years) }\end{array}$ & $\begin{array}{l}\text { Height } \\
\text { Mean } \pm \text { SD }(\mathrm{cm})\end{array}$ & $\begin{array}{l}\text { Weight } \\
\text { Mean } \pm \text { SD (kg) }\end{array}$ & $\begin{array}{l}\text { BMI Mean } \pm \text { SD } \\
\left(\mathrm{kg} / \mathrm{m}^{2}\right)\end{array}$ & Menopausal status Mean \pm SD \\
\hline $\operatorname{BCS}(n=16)$ & $52.0 \pm 4.0$ & $152.7 \pm 4.8$ & $71.1 * \pm 10.9$ & $30.6 \pm 4.97$ & $\begin{array}{l}\text { Post-menopausal within the previous } 12-84 \text { months } \\
(23.4 \pm 22.12 \text { months) }\end{array}$ \\
\hline CNT $(n=14)$ & $53.0 \pm 6.0$ & $162.6 \pm 7.8$ & $79.8^{*} \pm 9.7$ & $30.1 \pm 2.8$ & $\begin{array}{l}\text { Post-menopausal within the previous } 12-48 \text { months } \\
(14.9 \pm 9.6 \text { months) }\end{array}$ \\
\hline
\end{tabular}

* Significant different $(\mathrm{p}<0.05)$

Table 2 Peak torque (PT) and total work (TW) in breast cancer survivors and control group.

\begin{tabular}{|c|c|c|c|c|c|c|c|}
\hline & \multirow[b]{2}{*}{ SET } & \multicolumn{3}{|c|}{ Breast Cancer Survivors $(\mathrm{n}=16$ ) } & \multicolumn{3}{|c|}{ Control Group (n=14) } \\
\hline & & $1^{\text {st }}$ & $2^{\text {nd }}$ & $3^{\text {rd }}$ & $1^{\text {st }}$ & $2^{\text {nd }}$ & $3^{\text {rd }}$ \\
\hline \multirow[t]{2}{*}{ PT (N.m) } & $1 \mathrm{~min}$ & $107.6 \pm 19.9$ & $97.1 \pm 15.9^{\#}$ & $88.0 \pm 15.1^{*}$ & $133.4 \pm 20.8^{\S}$ & $118.9 \pm 19.6^{\# \S}$ & $108.9 \pm 17.2^{*}$ \\
\hline & $2 \mathrm{~min}$ & $106.8 \pm 16.7$ & $103.5 \pm 16.8^{\#} \dagger$ & $98.6 \pm 16.9 * \dagger$ & $135.3 \pm 17.1^{\S}$ & $124.3 \pm 17.0^{\#} \dagger^{\S}$ & $117.5 \pm 16.1^{*} \dagger$ \\
\hline \multirow[t]{2}{*}{ TW (J) } & $1 \mathrm{~min}$ & $897.4 \pm 175.0$ & $791.9 \pm 138.4^{\#}$ & $680.5 \pm 126.9^{*}$ & $1158.7 \pm 163.2^{\S}$ & $1009.1 \pm 163.1$ \#§ & $877.2 \pm 143.7 * \S$ \\
\hline & $2 \mathrm{~min}$ & $883.3 \pm 175.2$ & $832.2 \pm 152.0^{\#} \dagger$ & $786.6 \pm 146.0^{*} \dagger$ & $1171.7 \pm 159.4^{\S}$ & $1074.2 \pm 146.7^{\#} \dagger^{\S}$ & $980.7 \pm 188.0^{*} \dagger^{\S}$ \\
\hline
\end{tabular}

$\left({ }^{*}\right) \mathrm{p}<0.05$, lower than $1^{\text {st }}$ and $2^{\text {nd }}$ set

(\#) $p<0.05$, lower than $1^{\text {st }}$ set

(†) $\mathrm{p}<0.05$, higher than $1 \mathrm{~min}$

(§) Higher than breast cancer survivors 
Table 3 Decline (\%) of peak torque ( $\mathrm{Nm}$ ) between $1^{\text {st }}, 2^{\text {nd }}$ and $3^{\text {rd }}$ sets for breast cancer survivors and control group.

\begin{tabular}{|c|c|c|c|c|}
\hline & \multicolumn{2}{|c|}{ Breast cancer survivors $(n=16)$} & \multicolumn{2}{|c|}{ Control group $(n=14)$} \\
\hline & $1 \mathrm{~min}$ & $2 \mathrm{~min}$ & $1 \mathrm{~min}$ & $2 \mathrm{~min}$ \\
\hline $1^{\text {st }}$ vs. $2^{\text {nd }}$ set & $9.81 \%$ & $3.13 \%$ & $10.94 \%$ & $8.09 \%$ \\
\hline $2^{\text {nd }}$ vs. $3^{\text {rd }}$ set & $9.37 \%$ & $4.76 \%$ & $8.30 \%$ & $5.53 \%$ \\
\hline $1^{\text {st }}$ vs. $3^{\text {rd }}$ set & $18.27 \%$ & $7.74 \%$ & $18.33 \%$ & $13.17 \%$ \\
\hline
\end{tabular}

Table 4 Decline (\%) of total work (J) between $1^{\text {st }}, 2^{\text {nd }}$ and $3^{\text {rd }}$ sets for breast cancer survivors and control group.

\begin{tabular}{|c|c|c|c|c|}
\hline & \multicolumn{2}{|c|}{$\begin{array}{l}\text { Breast cancer survivors } \\
\qquad(n=16)\end{array}$} & \multicolumn{2}{|c|}{$\begin{array}{c}\text { Control group } \\
(n=14)\end{array}$} \\
\hline & $1 \mathrm{~min}$ & $2 \mathrm{~min}$ & $1 \mathrm{~min}$ & $2 \mathrm{~min}$ \\
\hline $1^{\text {st }}$ vs. $2^{\text {nd }}$ set & $11.76 \%$ & $5.80 \%$ & $12.91 \%$ & $8.31 \%$ \\
\hline $2^{\text {nd }}$ vs. $3^{\text {rd }}$ set & $14.07 \%$ & $5.47 \%$ & $13.07 \%$ & $8.70 \%$ \\
\hline $1^{\text {st }}$ vs. $3^{\text {rd }}$ set & $24.17 \%$ & $10.95 \%$ & $24.29 \%$ & $16.29 \%$ \\
\hline
\end{tabular}

TW of the knee extensor was significant greater in the CNT compared to the BCS group for all knee extension exercise sets $(p<0.001)$. There was a significant correlation between rest interval and exercise sets $(\mathrm{p}<0.001)$. TW was also higher in 2 min rest interval condition in the $2^{\text {nd }}$ and $3^{\text {rd }}$ set compared to 1 min rest condition for both groups $(\mathrm{p}<0.05)$. TW significantly decreased throughout exercise for both groups in both RI $(\mathrm{p}<0.05)$. The percent decline in PT and TW between $1^{\text {st }}, 2^{\text {nd }}$ and $3^{\text {rd }}$ sets were observed in both groups are presented in $\odot$ Table 3,4 , respectively.

\section{Discussion}

$\nabla$

Despite the ever-growing number of studies examining the effects of exercise in cancer patients, and the promising results reported by studies since the mid- $80 \mathrm{~s}$, many fundamental questions including what is the optimum mode, intensity, and frequency of exercise training for cancer patients remain unanswered. Until research addresses these fundamental questions, more specific exercise training guidelines cannot be devised.

Most recently, the use of resistance training has been explored as a mode of exercise designed to improve muscular fitness (i.e. development of strength) as well as provide positive changes in body composition [32]. However, in a recent systematic review of the literature on the effects of exercise training in breast cancer survivors, Battaglini et al. [5] reported that only 7 studies $[14,15,27,37,42-44]$ used resistance exercise as a mode of exercise training. Furthermore, just one study reported the RI used [42]. In this study [42], the authors used an RI between sets of 1 and $2 \mathrm{~min}$ following resistance training recommendations set forth by the American College of Sports Medicine exercise guidelines for healthy adults [34] and for older adults [2]. However, taking into consideration that CRF is a very common side effect experienced by breast cancer survivors who undergo chemotherapy and/or radiation therapy [16], and the potential negative effects of anti-cancer treatment on muscle physiology, it can be speculated that cancer patients may require a different RI between sets than that required for healthy adults or older men in order to maximize training responses and attain the best possible benefits from resistance training. There is scientific evi- dence regarding the benefits and safety of both resistance training and aerobic training administered to breast cancer survivors [36]; however, very few studies have provided detailed information on the resistance training regimens; more information is available on the RI between sets $[1,15,25,28,35]$. An adequate interim RI during resistance training is a very important variable that can be manipulated to characterize the objective of the training process, since it can affect metabolic, hormone, and cardiovascular responses to acute and chronic resistance training protocols [10]. Therefore, the purpose of this study was to compare the acute effect of 2 different RI between sets of knee extension exercise on muscle strength and muscle work capacity in breast cancer survivors and a control group. In this study, it was hypothesized that PT and TW in BCS would be lower than in the CNT group, and $1 \mathrm{~min}$ RI would be sufficient to recover PT and TW in the BCS group but not in the CNT group. This might be due to the possibility of the CNT group being able to produce higher PT than the BCS, which would require a longer $\mathrm{RI}$ in the CNT group to recover before the following exercise set. The results of the analyses showed a lower PT and TW in the BCS group when compared to the CNT group; however, surprisingly, one of the main findings of the present study was that a $2 \mathrm{~min}$ RI was not sufficient recovery time to allow both the BCS and CNT groups to maintain PT and TW during the 3 sets of exercise.

Differing from the results of the present study, Bottaro et al. [9] showed that in older men, a significant decline in PT between sets was observed when using $1 \mathrm{~min}$ RI, whereas there was no significant decline in PT with 2 min RI. The difference between our study and Bottaro's is an interesting finding and contradicted our hypothesis. Although the BCS participants in the present study produced low levels of force, they needed longer rest intervals than the older subjects, and this may be related to other physiological factors related to CRF [3]. Unfortunately, CRF was not assessed in the present study.

Theou et al. [40], also investigated the effects of 3 different RI (15, 30 and $60 \mathrm{~s}$ ) on PT and mean torque using 3 sets of 8 maximal isokinetic knee extension/flexion at $60^{\circ} . \mathrm{s}^{-1}$ in younger and older women. According to Theou and colleagues, the recovery time between sets of knee flexor exercise was faster among older women (30s) than younger women (60s). Theou and colleagues attributed the fast recovery in older women to the lighter loads they achieved during the exercise session. Although Theou et al. [40] also investigated postmenopausal women, their results are also not in agreement with the findings of the present study.

In another study by Bottaro and colleague [11], the researchers reported that $30 \mathrm{~s}$ RI between sets was also sufficient to minimize the reduced PT in older men. However, the protocol used in Bottaro and colleague's study [11] evaluated the effect of RI on isokinetic testing protocols by using 2 sets of 4 repetitions. Even though no direct comparisons can be made between Theou et al. [40] and Bottaro [9] studies to the current study, the results of the current study suggests that when older men and breast cancer survivors perform 10 repetitions of maximal isokinetic knee extension/flexion at $60^{\circ} . \mathrm{s}^{-1}$ a longer RI interval may be needed to minimize the decrease in PT between sets.

Repeated muscle contractions during resistance training result in muscular fatigue, usually expressed as a reduction in forceproducing capacity. Muscle fatigue response may be influenced by the manipulation of resistance training variables, among them RI [10]. In our study the TW was significantly greater in the CNT than in the BCS group for all knee extension exercise sets; TW was also higher in 2 min rest interval condition compared to 
1 min rest condition for both groups. The lower TW values observed in the BCS group may be associated with CRF derived from decreased physical activity levels during and post completion of anti-cancer treatment as well as the direct effect of chemotherapy and/or radiation treatments commonly administered to breast cancer patients. Furthermore, sleep disturbances, dietary intake alterations, and decreased muscle strength are all believed to contribute to the development of CRF which could significantly alter the BCS response to resistance training [3]. However, it is important to emphasize that this study was the first to evaluate the effect of RI between sets on muscle performance in breast cancer survivors, and therefore, more research is needed to confirm or refute the results of this current study. Unfortunately, due to some study limitations, generalization of the results cannot be made at this time. However, this current study can be seen as hypothesis-generating, providing initial information necessary for the development of future studies examining the acute responses of resistance training in breast cancer survivors. For future research, it is therefore recommended that a larger sample size, different RI intervals, assessment of CRF, and the evaluation of other muscle groups be taken into account. Since all muscles do not appear to be affected in a similar manner either qualitatively and quantitatively, and changes in different muscles may be influenced by the aging process [26] and in breast cancer survivors, the potential influence of anti-cancer treatments and reduced levels of physical activity on muscle integrity [4] should be studied.

\section{Conclusion}

$\nabla$

The results of this study suggest that breast cancer survivors may need a longer rest interval (longer than $2 \mathrm{~min}$ ) to better recover between sets, thus minimizing the drop in PT and TW during 3 sets of 10 repetitions of maximal isokinetic knee extension/flexion at $60^{\circ} . \mathrm{s}^{-1}$. Translating the results of this study into practice, researchers and strength and conditioning professionals may begin to explore different RI intervals when working with breast cancer survivors as a means to allow BCS to complete a strength training session while maximizing their proposed training goals.

\section{Acknowledgements}

$\nabla$

This study was supported by CAPES (process 99999.011833/ 2013-00).

Conflict of interest: The authors have no conflict of interest to declare.

\footnotetext{
Affiliations

${ }^{1}$ College of Physical Education, Federal University of Goias, Goiania, Brazil ${ }^{2}$ Department of Exercise and Sport Science and Lineberger Comprehensive Cancer Center, University of North Carolina at Chapel Hill, Chapel Hill, United States

${ }^{3}$ College of Physical Education, University of Brasilia, Brasilia, Brazil

${ }^{4}$ Medical College Federal University of Brasilia, Brazil

${ }^{5}$ Medical College, Federal University of Goias, Goiania, Brazil

${ }^{6}$ Faculty of Health Sciences, University of Brasilia, Brasilia, Brazil
}

\section{References}

1 Ahmed RL, Thomas W, Yee D, Schmitz KH. Randomized controlled trial of weight training and lymphedema in breast cancer survivors. J Clin Oncol 2006; 24: 2765-2772

2 American College of Sports M, Chodzko-Zajko WJ, Proctor DN, Fiatarone Singh MA, Minson CT, Nigg CR, Salem GJ, Skinner JS. American College of Sports Medicine position stand. Exercise and physical activity for older adults. Med Sci Sports Exerc 2009; 41: 1510-1530

3 Battaglini C, Dennehy C, Groff D, Kirk D, Anton P. Complementary therapies in the management of cancer treatment-related symptoms: The individualized prescriptive exercise intervention approach. Med Sport 2006; 10: 8

4 Battaglini CL, Hackney AC, Goodwin ML. Cancer cachexia: muscle physiology and exercise training. Cancers 2012; 4: 1247-1251

5 Battaglini CL, Mills RC, Phillips BL, Lee JT, Story CE, Nascimento MGB, Hackney AC. Twenty-five years of research on the effects of exercise training in breast cancer survivors: A systematic review of the literature. World J Clin Oncol 2014; 5: 13

6 Bilodeau M, Erb MD, Nichols JM, Joiner KL, Weeks JB. Fatigue of elbow flexor muscles in younger and older adults. Muscle Nerve 2001; 24: 98-106

7 Bilodeau M, Henderson TK, Nolta BE, Pursley PJ, Sandfort GL. Effect of aging on fatigue characteristics of elbow flexor muscles during sustained submaximal contraction. J Appl Physiol 2001; 91: 2654-2664

8 Bottaro M, Brown LE, Celes R, Martorelli S, Carregaro R, de Brito Vidal $J C$. Effect of rest interval on neuromuscular and metabolic responses between children and adolescents. Pediatr Exerc Sci 2011; 23: 311-321

9 Bottaro M, Ernesto C, Celes R, Farinatti PT, Brown LE, Oliveira RJ. Effects of age and rest interval on strength recovery. Int J Sports Med 2010; 31: $22-25$

10 Bottaro M, Martins B, Gentil P, Wagner D. Effects of rest duration between sets of resistance training on acute hormonal responses in trained women. J Sci Med Sport 2009; 12: 73-78

11 Bottaro $M$, Russo $A F$, de Oliveira RJ. The effects of rest interval on quadriceps torque during an isokinetic testing protocol in elderly. J Sports Sci Med 2005; 4: 285-290

12 Celes R, Brown LE, Pereira MC, Schwartz FP, Junior VA, Bottaro M. Gender muscle recovery during isokinetic exercise. Int J Sports Med 2010; 31: 866-869

13 Clark BC, Collier SR, Manini TM, Ploutz-Snyder LL. Sex differences in muscle fatigability and activation patterns of the human quadriceps femoris. Eur J Appl Physiol 2005; 94: 196-206

14 Cormie P, Pumpa K, Galvão DA, Turner E, Spry N, Saunders C, Zissiadis $Y$, Newton $R U$. Is it safe and efficacious for women with lymphedema secondary to breast cancer to lift heavy weights during exercise: a randomised controlled trial. J Cancer Surviv 2013; 7: 11

15 Courneya KS, Segal RJ, Mackey JR, Gelmon K, Reid RD, Friedenreich CM, Ladha AB, Proulx C, Vallance JK, Lane K, Yasui Y, McKenzie DC. Effects of aerobic and resistance exercise in breast cancer patients receiving adjuvant chemotherapy: a multicenter randomized controlled trial. J Clin Oncol 2007; 25: 4396-4404

16 Curt GA, Breitbart W, Cella D, Groopman JE, Horning SJ, Itri LM, Johnson DH, Miaskowski C, Scherr SL, Portenoy RK, Vogelzang NJ. Impact of cancer-related fatigue on the lives of patients: new findings from the Fatigue Coalition. Oncologist 2000; 5: 353-360

17 De Backer IC, Schep G, Backx FJ, Vreugdenhil G, Kuipers H. Resistance training in cancer survivors: a systematic review. Int J Sports Med 2009; 30: 703-712

18 de Salles BF, Simao R, Miranda F, Novaes Jda S, Lemos A, Willardson $J M$. Rest interval between sets in strength training. Sports Med 2009; 39: 765-777

19 Ditor DS, Hicks AL. The effect of age and gender on the relative fatigability of the human adductor pollicis muscle. Can J Physiol Pharmacol 2000; 78: 781-790

20 Harriss DJ, Atkinson G. Ethical standards in sport and exercise science research: 2014 update. Int J Sports Med 2013; 34: 1025-1028

21 Hicks AL, McCartney N. Gender differences in isometric contractile properties and fatigability in elderly human muscle. Can J Appl Physiol 1996; 21: 441-454

22 Hunter SK, Critchlow A, Enoka RM. Influence of aging on sex differences in muscle fatigability. J Appl Physiol 2004; 97: 1723-1732

23 Katsiaras A, Newman AB, Kriska A, Brach J, Krishnaswami S, Feingold E, Kritchevsky SB, Li R, Harris TB, Schwartz A, Goodpaster BH. Skeletal muscle fatigue, strength, and quality in the elderly: the Health $A B C$ Study. J Appl Physiol 2005; 99: 210-216 
24 Lanza IR, Russ DW, Kent-Braun JA. Age-related enhancement of fatigue resistance is evident in men during both isometric and dynamic tasks. J Appl Physiol 2004; 97: 967-975

25 Ligibel JA, Campbell N, Partridge A, Chen WY, Salinardi T, Chen H, Adloff $K$, Keshaviah A, Winer EP. Impact of a mixed strength and endurance exercise intervention on insulin levels in breast cancer survivors. J Clin Oncol 2008; 26: 907-912

26 Macaluso A, De Vito G. Muscle strength, power and adaptations to resistance training in older people. Eur J Appl Physiol 2004; 91: 450-472

27 Musanti R. A study of exercise modality and physical self-esteem in breast cancer survivors. Med Sci Sports Exerc 2012; 44: 352-361

28 Ohira T, Schmitz KH, Ahmed RL, Yee D. Effects of weight training on quality of life in recent breast cancer survivors: the Weight Training for Breast Cancer Survivors (WTBS) study. Cancer 2006; 106: 2076-2083

29 OMS. Investigaciones sobre la menopausia en los años noventa. In: Salud OMdl. ed. Genebra, 1996

30 Parcell AC, Sawyer RD, Tricoli VA, Chinevere TD. Minimum rest period for strength recovery during a common isokinetic testing protocol. Med Sci Sports Exerc 2002; 34: 1018-1022

31 Pincivero DM, Gandaio CM, Ito Y. Gender-specific knee extensor torque, flexor torque, and muscle fatigue responses during maximal effort contractions. Eur J Appl Physiol 2003; 89: 134-141

32 Pincivero DM, Lephart SM, Karunakara RG. Effects of rest interval on isokinetic strength and functional performance after short term high intensity training. Br J Sports Med 1997; 31: 6

33 Pincivero DM, Lephart SM, Karunakara RG. Effects of intrasession rest interval on strength recovery and reliability during high intensity exercise. J Strength Cond Res 1998; 12: 5

34 Ratamess NA, Alvar BA, Evetoch TK, Housh TJ, Kibler WB, Kraemer WJ, Triplett NT. American College of Sport Medicine position stand. Progression Models in resistance training for health adults. Med Sci Sports Exerc 2009; 41: 21
35 Schmitz KH, Ahmed RL, Hannan PJ, Yee D. Safety and efficacy of weight training in recent breast cancer survivors to alter body composition, insulin, and insulin-like growth factor axis proteins. Cancer Epidemiol, Biomarkers Prev 2005; 14: 1672-1680

36 Schmitz KH, Courneya KS, Matthews C, Demark-Wahnefried W, Galvao $D A$, Pinto BM, Irwin ML, Wolin KY, Segal RJ, Lucia A, Schneider CM, von Gruenigen VE, Schwartz AL, American College of Sports M. American College of Sports Medicine roundtable on exercise guidelines for cancer survivors. Med Sci Sports Exerc 2010; 42: 1409-1426

37 Schwartz AL, Winters-Stone K, Gallucci B. Exercise effects on bone mineral density in women with breast cancer receiving adjuvant chemotherapy. Oncol Nurs Forum 2007; 34: 627-633

38 Sforzo GA, Touey PR. Manipulating exercise order affects muscular performance during a resistance exercise training session. J Strength Cond Res 1996; 10: 4

39 Stumbo TA, Merriam S, Nies K, Smith A, Spurgeon D, Weir JP. The effect of hand-grip stabilization on isokinetic torque at the knee. J Strength Cond Res 2001; 15: 372-377

40 Theou 0 , Gareth JR, Brown LE. Effect of rest interval on strength recovery in young and old women. J Strength Cond Res 2008; 22: 6

41 Touey PR, Sforzo GA, McManis BG. Effect of manipulating of rest periods on isokinetic muscle performance. Med Sci Sports Exerc 1994; 26

42 Winters-Stone KM, Dobek J, Bennett JA, Nail LM, Leo MC, Schwartz A. The effect of resistance training on muscle strength and physical function in older, postmenopausal breast cancer survivors: a randomized controlled trial. J Cancer Surviv 2012; 6: 189-199

43 Winters-Stone KM, Dobek J, Nail L, Bennett JA, Leo MC, Naik A, Schwartz A. Strength training stops bone loss and builds muscle in postmenopausal breast cancer survivors: a randomized, controlled trial. Breast Cancer Res Treat 2011; 127: 447-456

44 Winters-Stone KM, Dobek J, Nail LM, Bennett JA, Leo MC, TorgrimsonOjerio B, Luoh SW, Schwartz A. Impact+resistance training improves bone health and body composition in prematurely menopausal breast cancer survivors: a randomized controlled trial. Osteoporos Int 2013; 24: $1637-1646$ 